\title{
MONTE-CARLO BASED UNCERTAINTY ANALYSIS: SAMPLING EFFICIENCY AND SAMPLING CONVERGENCE
}

\author{
HANS JANSSEN \\ Building Physics Section \\ Department of Civil Engineering \\ KU Leuven \\ Kasteelpark Arenberg 40 \\ 3000 Leuven \\ Belgium \\ email: hans.janssen@bwk.kuleuven.be \\ tel: +3216321326
}

\begin{abstract}
Monte Carlo analysis has become nearly ubiquitous since its introduction, now over 65 years ago. It is an important tool in many assessments of the reliability and robustness of systems, structures or solutions. As the deterministic core simulation can be lengthy, the computational costs of Monte Carlo can be a limiting factor. To reduce that computational expense as much as possible, sampling efficiency and convergence for Monte Carlo are investigated in this paper. The first section shows that non-collapsing spacefilling sampling strategies, illustrated here with the maximin and uniform Latin hypercube designs, highly enhance the sampling efficiency, and render a desired level of accuracy of the outcomes attainable with far lesser runs. In the second section it is demonstrated that standard sampling statistics are inapplicable for Latin hypercube strategies. A sample-splitting approach is put forward, which in combination with a replicated Latin hypercube sampling allows assessing the accuracy of Monte Carlo outcomes. The assessment in turn permits halting the Monte Carlo simulation when the desired levels of accuracy are reached. Both measures form fairly noncomplex upgrades of the current state-of-the-art in Monte-Carlo based uncertainty analysis but give a substantial further progress with respect to its applicability.
\end{abstract}

\section{KEYWORDS}

Monte Carlo, uncertainty analysis, space-filling Latin hypercube, sampling efficiency, sampling convergence, sample-splitting

\section{HighLIGHTS}

- Monte Carlo is virtually universal, but computational expense is important barrier

- A good sampling strategy and convergence assessment will improve applicability

- Space-filling Latin hypercube designs are most efficient, should be generally used

- Sample-splitting on replicated Latin hypercube designs allows assessing accuracy

- Both measures are undemanding upgrades, but substantially boost the applicability 
Postprint: Janssen H. 2013. Monte-Carlo based uncertainty analysis: Sampling efficiency and sampling convergence, Reliability Engineering \& System Safety, 109: 123-132.

doi: $10.1016 /$ j.ress.2012.08.003

\section{INTRODUCTION}

The importance of identifying, characterising and displaying the uncertainties on the results of analyses of complex systems is progressively more recognised: many regulatory standards and guidelines explicitly demand an uncertainty appraisal as part of a performance assessment of structures, systems and solutions [1]. This appraisal of uncertainties is naturally connected to the concepts of 'reliability' and 'robustness'. Reliability can be defined as the probability for a solution to function without failure during a given interval of time, while robustness can be described as the persistence of the characteristic behaviour of a solution under uncertain conditions. Reliability, in essence, focuses on the probability of failure, while robustness more generally targets the probability of a certain performance level. But both concepts fundamentally require the assessment of probabilities, calling for the application of probabilistic methodologies rather than deterministic techniques. Or, quoting Oberkampf and co-authors, 'realistic modelling and simulation of complex systems must include the non-deterministic features of the system and the environment' [2].

Sampling-based uncertainty analysis, via Monte Carlo approaches, plays a central role in this characterisation and quantification of uncertainty [3]. In reliability, three methodology levels are commonly distinguished [4]. Level I techniques only evaluate whether the reliability is sufficient or not, without quantifying the actual failure probability. Level II techniques yield a simplified estimate of the failure probability via first and second order reliability methods. Level III techniques, finally, accurately calculate the failure probability, from exact probability density functions and limit state equations. In this class, Monte-Carlo based uncertainty analysis is taken to be the most dependable technique, as it is widely applicable - certain guidelines and limitations are to be considered however [5]-[7]. An analogous formalism is not in place yet for robustness assessment, but similar inferences can be made: at the highest level quantification of the spread on the performance of structures, systems or solutions is desired, and Monte-Carlo based uncertainty analysis similarly forms a frequently employed procedure.

As a result applications of Monte-Carlo-based uncertainty analysis can now be found in virtually all engineering fields: from the study of electronic circuits [8], over the design of built structures [9], to the appraisal of nuclear waste sites [10]. Several other disciplines also use Monte-Carlo based uncertainty analysis [11]: selected evocative examples are health care optimisation [12], economic risk evaluation [13], and defence scenario comparison [14]. Monte-Carlo-based uncertainty analysis likewise takes on a progressively increasing share in generic reliability and robustness assessments, via its task in static [15] and dynamic [16] fault tree analyses. Monte-Carlo methods are finally also applied as numerical integration methods [17]. All in all hence, in probabilistic modelling and simulation, Monte Carlo appears nearly universal, and progressively so.

The same is true for probabilistic analysis within building simulation. A reliable assessment of heat and mass transport in building materials, building components and whole buildings is central to reaching a sustainable, durable, healthy and comfortable built environment [18]-[21]. In this field of building design and diagnosis, application of deterministic building simulation models can currently be considered as state-of-the-art.

Most of the related parameters - material properties, boundary conditions, human actions - are however best characterised as stochastic variables. Probabilistic modelling hence was introduced within building simulation over two decades ago [22],[23] and is current-ly taking a high flight [24]-[30]. Three main approaches to inserting the 'uncertainties of system and environment' in building simulation models are in use [24]: stochastic differential equations [22], first/second order reliability methods [26], and Monte Carlo sampling-based techniques [27]. While the first two decidedly have potential, Monte Carlo currently prevails due to the complex, non-linear and transient character of most building simulation analyses [28]-[30]. 
Postprint: Janssen H. 2013. Monte-Carlo based uncertainty analysis: Sampling efficiency and sampling convergence, Reliability Engineering \& System Safety, 109: 123-132.

doi: $10.1016 /$ j.ress.2012.08.003

In conventional Monte-Carlo-based uncertainty analysis a series of deterministic calculations is executed, each with differing input values - sampled from their respective distributions - to obtain probability distributions of the targeted outcomes. Monte Carlo is hence inherently computationally expensive, particularly when the involved deterministic calculation requires much computing time [31]. In that respect a minimisation of the number of required Monte Carlo runs is essential. This can be obtained through a twoprong approach: sampling designs should be highly efficient, and sampling convergence should be reliably quantified. The latter permits halting the Monte Carlo analysis once suitably accurate outcomes have been reached, the former allows attaining such desired accuracy levels with a minimal number of simulation runs. And while Monte Carlo is already past its $65^{\text {th }}$ birthday [11], there appears to be room for improvement on both fronts. Latin hypercube sampling has advanced sampling efficiency [32] but further optimisations currently remain unassessed [33]-[37]. Concerning convergence monitoring, the achievements are scarce and fragmentary [38]-[42], and they moreover do not easily apply to advanced sampling designs. This paper addresses both issues, via an empirical application on a building simulation benchmark case. It is finally remarked here that this paper focuses on robustness rather than reliability, or on the general uncertainty of analysis outcomes rather than the low probability of failure events. This means that universal exploratory sampling designs are assessed while low probability sampling strategies - like importance sampling or subset simulation - are not dealt with.

The paper is built up as follows. After a concise synthesis of the literature, the two introductory sections put forward the stochastic calculation object and the selected sampling strategies. Their sampling efficiency is assessed in the first core section, showing major performance gains for the space-filling Latin hypercube designs. The second core section combines replicated Latin hypercube designs with sample-splitting bootstrapping to monitor sampling convergence. A closing example further demonstrates the benefits of improving sampling efficiency and monitoring sampling convergence.

\section{LITERATURE REVIEW}

Sampling efficiency and sampling convergence for Monte-Carlo based uncertainty analysis have been addressed earlier. A concise synthesis of the relevant literature allows to better set the stage for this paper.

\subsection{Sampling efficiency}

The concept of 'sampling efficiency' can be best described as the number of simulation runs required to bring the variance on the targeted outcomes below a preset level, or in other words, to obtain a certain level of accuracy. Sampling strategies that require less runs are termed 'more efficient'. Efficient sampling strategies are thus needed for maximal reduction of the computational expense of Monte Carlo analyses.

A well known variance-reduction technique in Monte Carlo is Latin hypercube sampling, whose superiority over random sampling has been corroborated often already. A theoretical analysis of the performance of Latin hypercube and basic random sampling was brought first by McKay and co-authors [32]: they found that Latin hypercube sampling is more efficient than random sampling when the desired output is monotonic in all inputs. Stein [43] improved on that by deriving that a single input having a non-zero main effect on the output is a sufficient condition for the superior efficiency of Latin hypercube sampling. Helton and Davis [44]-[45] made a wide-ranging applied investigation, based on a series of abstract problems. They observed that Latin hypercube sampling mostly outperforms random sampling, also for non-monotonic problems. By now, that conclusion has been confirmed with a variety of other applications [46]-[49]. 
Postprint: Janssen H. 2013. Monte-Carlo based uncertainty analysis: Sampling efficiency and sampling convergence, Reliability Engineering \& System Safety, 109: 123-132.

doi: $10.1016 /$ j.ress.2012.08.003

The introduction of Latin hypercube sampling has been succeeded by further optimisations [33]-[37], which usually target a better space-filling character. Extensive overviews can be found in [50]-[54], two popular examples are the 'maximin' and 'uniform' Latin hypercube designs [50],[51]. To the author's knowledge of the author, no actual evaluation of their sampling efficiency relative to normal Latin hypercube sampling has been made to date. Several authors do consider maximin and uniform designs when evaluating the performance of metamodels [34],[55],[56], but the influences of the metamodelling method and the sampling strategy cannot be separated. In this paper hence, such evaluation of the sampling efficiency of basic random versus standard, maximin and uniform Latin hypercube sampling designs is executed.

\subsection{Sampling convergence}

Besides use of efficient sampling designs, the ability to halt the analysis when sufficient accuracy has been reached equally allows reducing the calculation time of Monte Carlo simulations. The possibility to sequentially add sampling runs and appropriate monitoring of the sampling convergence are hence also important for maximal reduction of the computational expense of Monte Carlo analyses.

For random sampling, the continuous addition of simulation runs is evident. When only the mean of the targeted outcome is relevant, error estimates based on the variance on the mean - the variance on the targeted outcome divided by the current number of runs - can be used in a convergence criterion [38], an approach often applied in commercial packages [57]-[60]. For aggregated results - percentiles, distributions, ... - this direct error approach does not work though. Batch statistics can be applied instead, as is mainly done in nuclear engineering and in radiological sciences [39]-[42]. The relevant set of outcomes is divided to subsets, the aggregated result is quantified for each subset, and the variance on these aggregated results is derived. This method is very much parallel to 'sample-splitting' bootstrap methods, very common in general statistics [61][64].

These techniques are not directly applicable for Latin hypercube designs however. The number of runs in a Latin hypercube sampling design is generally predetermined, albeit that methods to extend Latin hypercube designs are being proposed [65],[66]. Random-sampling-based error estimates via the variance on the mean are furthermore not valid for Latin hypercube sampling. A single Latin hypercube can in addition not be correctly split into subsets, since this would nullify the specific sampling design. Replicated Latin hypercubes have been applied though to make a-posteriori accuracy estimates [39],[40]. These replicates all were complete and self-sufficient sampling designs though and the aim was to confirm the stability of the analysis outcomes. In this paper, the concepts of replicated Latin hypercubes and sample-splitting bootstrapping are combined, to obtain the desired convergence monitoring and sequential addition for Latin hypercube sampling designs.

\section{Calculation object}

The fourth benchmark from in the HAMSTAD project [67] is taken as exemplary 'calculation object' in this study. The HAMSTAD project formulated five benchmark simulation exercises, for comparative testing of simulation models for heat and moisture transfer in building components. The fourth benchmark quantifies the hygrothermal response of a construction consisting of $100 \mathrm{~mm}$ of ceramic brick and $20 \mathrm{~mm}$ of interior finish, to variations in both the interior and the exterior boundary conditions over a period of five days. Further info on this benchmark exercise can be found in [67]-[68]. All simulations are made with a numerical model for heat and moisture transfer in building components [69]-[72]. 
Postprint: Janssen H. 2013. Monte-Carlo based uncertainty analysis: Sampling efficiency and sampling convergence, Reliability Engineering \& System Safety, 109: 123-132.

doi: $10.1016 /$ j.ress.2012.08.003

While the original benchmark quantifies temperature and moisture content fields in space and time, this analysis restricts the outcome to the cumulated exchange of heat and moisture between the interior environment and construction. In other words, simulation targets are the cumulated heat losses from, and the cumulated moisture gains to the interior environment. These are quantified by cumulating respectively the convective and radiative heat flows from interior zone to interior surface and the convective vapour flow from interior surface to interior zone:

$$
\begin{aligned}
& H L=\int_{0}^{5 d} q_{i \rightarrow s i}(\tau) d \tau=\int_{0}^{5 d} \alpha_{i}\left[T_{i}(\tau)-T_{s i}(\tau)\right] d \tau \\
& M G=\int_{0}^{5 d} g_{v, s i \rightarrow i}(\tau) d \tau=\int_{0}^{5 d} \beta_{i}\left[p_{v, s i}(\tau)-p_{v, i}(\tau)\right] d \tau
\end{aligned}
$$

in which $H L\left(\mathrm{~J} / \mathrm{m}^{2}\right)$ are cumulated heat losses, $M G\left(\mathrm{~kg} / \mathrm{m}^{2}\right)$ cumulated moisture gains, $T$ $(\mathrm{K})$ temperature, $p_{v}(\mathrm{~Pa})$ partial vapour pressure, $q\left(\mathrm{~W} / \mathrm{m}^{2}\right)$ heat flux, $g_{v}\left(\mathrm{~kg} / \mathrm{m}^{2} \cdot \mathrm{s}\right)$ vapour flux, $\alpha\left(\mathrm{W} / \mathrm{m}^{2} \cdot \mathrm{K}\right)$ surface heat transfer coefficient, $\beta\left(\mathrm{kg} / \mathrm{m}^{2} \cdot \mathrm{s} \cdot \mathrm{Pa}\right)$ surface vapour transfer coefficient, $T$ (s) time, and subscripts $i /$ si signify interior environment/interior surface. All HAMSTAD benchmarks are originally deterministic, and the probabilistic component hence needs to be specifically introduced. For this academic application, two brick material properties and two surface transfer coefficients are transformed into aleatory variables with uniform distributions: the brick's capillary absorption coefficient may take values from 0.05 to $0.35 \mathrm{~kg} / \mathrm{m}^{2} \cdot \mathrm{s}^{0.5}$, the brick's thermal conductivity from 0.5 to $1.1 \mathrm{~W} / \mathrm{m} \cdot \mathrm{K}$, the interior surface heat transfer coefficient from 5 to $11 \mathrm{~W} / \mathrm{m}^{2} \cdot \mathrm{K}$, and the exterior surface moisture transfer coefficient from $5 \cdot 10^{-8}$ to $35 \cdot 10^{-8} \mathrm{~kg} / \mathrm{m}^{2} \cdot \mathrm{s} \cdot \mathrm{Pa}$. And while these four stochastic variables may not all explicitly represented in the $\mathrm{HL}$ and $M G$ expressions in equation (1), they all do affect the $q$ and $g_{v}$, via the transfer properties or boundary conditions [69]. Their ranges are determined such that all four variables have a significant impact on the benchmark outcomes while staying within acceptable physical limits.

\section{SAMPLING STRATEGIES}

A Monte Carlo analysis basically aims at performing a sufficiently large sequence of simulations $n$, each with different values for the $k$ input parameters, to dependably quantify the probability distributions of the targeted outcomes. Monte Carlo hence requires selecting $n$ samples out of each of the $k$ input parameter distributions. Generally, the $n$ k-tuples are first drawn from uniform distributions in the k-dimensional hypercube $[0,1]^{\mathrm{k}}$ and afterwards transformed to a set of $n$ samples satisfying the probability distributions of the $\mathrm{k}$ separate input parameters. Two major criteria for sampling designs are put forward in [50],[51]: they should be 'space-filling' and 'non-collapsing'. Without prior info on the functional behaviour of the outcomes, it is essential to obtain results for the full multi-parameter space (or k-dimensional hypercube). Sampling points should therefore be evenly distributed, or 'space-filling'. Moreover, without prior info on the relative weights of the different parameters (or dimensions), it is vital that no two design points coincide when projected onto a lower number of dimensions, or 'non-collapsing'.

Basic random sampling (BRS) makes use of standard pseudo-random numbers, from a random-number generator. Basic random sampling is relatively simple but computationally expensive, as many runs are needed to sample the multi-parameter space in a representative way. This is illustrated in Figure 1 (top left), depicting BRS sampling points for 10 runs and 2 input variables. The sampling points are shown with their root values in the two-dimensional hypercube, before being transformed to actual probability distributions thus. BRS is obviously neither space-filling nor non-collapsing.

Latin hypercube sampling improves on the 'non-collapsing' part: each input distribution is divided into $n$ equiprobable strata and each stratum is sampled randomly once. LHS 
Postprint: Janssen H. 2013. Monte-Carlo based uncertainty analysis: Sampling efficiency and sampling convergence, Reliability Engineering \& System Safety, 109: 123-132.

doi: $10.1016 /$ j.ress.2012.08.003

designs are fairly easy to generate: $\mathrm{k}$ random permutations of integers 1 to $\mathrm{n}$ designate the strata to be sampled for each input parameter, complemented by random sampling in each selected stratum. Figure 1 (top right) shows that LHS indeed gives non-collapsing designs. That does not automatically translate into a space-filling design however: frequently correlations (for low k) and clusters (for high k) appear [73],[74]. This is shown in Figure 1 (top right) as well: the uniformity of the sampling points in the multiparameter space is far from optimal. These spurious correlations in Latin hypercube can be remedied [73],[74], but such corrections are unfortunately very often not put to use [47]-[49].

Many different criteria are available in relation to the space-filling character of sampling designs: extensive overviews can be found in [50]-[54]. To maintain the 'non-

collapsing' property, only space-filling Latin hypercube designs are considered in this study. It has extensively been shown that space-filling designs without Latin hypercube requirement put fairly many sampling points near the edges of the multi-parameter space [34],[50],[51], [55], which is undesirable.

For the study here, two common space-filling criteria are retained: 'distance-based' and 'uniformity-based' designs [50],[51]. In distance-based sampling designs multi-dimensional distances between the sampling points are optimised, with maximin and minimax as best-known criteria [33]. Maximin sampling designs maximise the minimal distance between sampling points, minimax designs minimise the maximal distance; here the maximin criterion is applied (DIS). In uniformity-based sampling designs, the discrepancy of the set of sampling points - the global deviation from a uniform sampling point density - is minimised, with the centred and wrap-around discrepancy as best-known criteria [35]. Both discrepancies do not only assess the uniformity of the $n$ sampling points within the k-dimensional hypercube $[0,1]^{\mathrm{k}}$ but also the uniformity of all projections on a lower number of dimensions; here the centred discrepancy is used (UNI).

The space-filling designs applied here are hence maximin and centred-discrepancy optimised LHS designs. Generating such space-filling design is, contrary to BRS or LHS, a daunting task: this type of combinatorial optimisation problems is known as 'NP-hard problems', growing exponentially with the size of the sampling design [51]. For the designs here the enhanced stochastic evolutionary algorithm of Jin and co-authors [75] is employed. Examples of the DIS and UNI designs are shown in Figure 1 (bottom), illustrating their space-filling and non-collapsing properties. It should, in that respect, be noted that space-filling Latin hypercube designs use midpoint sampling in the equiprobable strata, in contrast to random sampling in standard Latin hypercube designs.

\section{SAMPLING EFFICIENCY}

This section assesses the sampling efficiency of the random (BRS) and Latin hypercube (LHS, DIS, UNI) sampling strategies by comparing their deviations from a reference solution at equal number of runs. For each strategy, four-parameter sampling designs are first generated in the $[0,1]^{4}$ hypercube: these pointer variables are then converted to the uniform distributions of the actual uncertain variables put forward in section 3 .

\subsection{Reference solution}

A 100000-run BRS Monte Carlo analysis serves as the reference solution, the basis for comparison of all other solutions. The resulting cumulative distributions for $\mathrm{HL}$ and $\mathrm{MG}$ are shown in Figure 2. For the remainder of the paper they are characterised with their mean $\left(\mu_{\mathrm{HL}}, \mu_{\mathrm{MG}}\right)$ and standard deviation $\left(\sigma_{\mathrm{HL}}, \mu_{\mathrm{MG}}\right)$, which are collected in Table 1 . It can be shown that similar conclusions are reached when employing other criteria, for example median and interpercentile range, or the overall shape of the probability distribution. Figure 2 and Table 1 demonstrate the effect of the uncertain input parameters: a broad range of variation is observed for both $\mathrm{HL}$ and $\mathrm{MG}$. 
Postprint: Janssen H. 2013. Monte-Carlo based uncertainty analysis: Sampling efficiency and sampling convergence, Reliability Engineering \& System Safety, 109: 123-132.

doi: $10.1016 / \mathrm{j}$. ress.2012.08.003

Table 1 contains the resulting reference $\mu$ and $\sigma$ values, deduced from that 100000-run solution. To assess their accuracy, standard deviations on the value are quantified, via sampling statistics [76] and bootstrapping [61],[62]. Sampling statistics suggests that:

$$
\begin{aligned}
& \operatorname{std}(\mu)=\sigma \sqrt{\frac{1}{n}} \\
& \operatorname{std}(\sigma)=\frac{\sigma}{2} \sqrt{\frac{2}{n-1}+\frac{\kappa}{n}}
\end{aligned}
$$

where $n$ is the number of runs, $\mu$ the mean of the n-run analysis, $\sigma$ the standard deviation of the n-run analysis, $\kappa$ the excess kurtosis of the n-run analysis, and std are the expected standard deviations on $\mu$ and $\sigma$. Equation (2) hence implies that the accuracy of random-sampling-based Monte Carlo analysis depends on the probability distribution of the outcome and on the number of runs. The expected standard deviations can equally be obtained with bootstrapping: the original 100000 results are resampled (with replacement) for 100 times and means and standard deviations are computed for all resampled set. Standard deviations on these are equivalent to the std's obtained with equation (2). The standard deviations are finally normalised by division with $\mu$, to get a relative deviation. Both $\operatorname{std}(\mu)$ and $\operatorname{std}(\sigma)$ are indeed normalised by dividing by $\mu$ to come to a similar perspective on the accuracy of $\mu$ and $\sigma$.

Results from the two approaches are collected in Table 1. Overall the normalised standard deviations hover around $0.05 \%$ for $\mathrm{HL}$ and $0.1 \%$ for $\mathrm{MG}$, with decent agreements between the statistics and bootstrapping results. Such normalised standard deviations represent the accuracy of the obtained $\mu$ and $\sigma$ and can hence be taken as the lower limit when assessing other Monte Carlo solutions. They are assessed on their ability to estimate $\mu_{\mathrm{HL}}, \sigma_{\mathrm{HL}}, \mu_{\mathrm{MG}}$ and $\sigma_{\mathrm{MG}}$, and there is hence no need for additional runs in a samling design once similar deviations from the reference solution are reached.

\subsection{Random sampling}

The sampling efficiency of BRS is evaluated for $10,20,50,100,250$ and 500 runs. To account for stochastic variations in the generation of random numbers, every $n$-run set is repeated ten times, considered as 'one series' here. To obtain an even broader perspective, three series of ten repetitions of every n-run set are performed. The BRS results are collected in Figure 3, comparing the BRS prediction of $\mu$ and $\sigma$ to the reference solution. It is obvious that all BRS results hover around the reference solutions and that the deviations from the reference solution reduce progressively with an increasing number of runs $n$, as predicted by equation (2).

The same results are also shown in Figure 4, but now shown as normalised deviations from the reference solution (for every distinct $n$-run solution) and their root-mean-square averages (over a series of ten $n$-run solutions):

$$
\begin{aligned}
& \text { norm. deviat. }=\frac{\mid \mu_{\mathrm{i}} \text { or } \sigma_{\mathrm{i}}-\mu_{\mathrm{ref}} \text { or } \sigma_{\text {ref }} \mid}{\mu_{\text {ref }}} \\
& \text { norm. RMSD }=\sqrt{\frac{\sum_{10}(\text { nor. deviat. })^{2}}{10}}
\end{aligned}
$$

in which norm. deviat. is the normalised deviation for each solution, norm. RMSD is the overall normalised root-mean-square deviation, $\mu / \sigma_{\text {ref }}$ the reference values for $\mu / \sigma$, and $\mu / \sigma_{i}$ the solution for the $\mathrm{i}^{\text {th }}$ repetition in the series. The norm. RMSD are of course equal to the normalised standard deviations on $\mu$ and $\sigma$, and they hence represent the 'average relative deviation' of all n-run Monte-Carlo solutions. In other words, they represent the accuracy of the n-run Monte-Carlo results in relation to the reference solution. Further in this paper, results are often only shown as normalised root-mean-square devia- 
Postprint: Janssen H. 2013. Monte-Carlo based uncertainty analysis: Sampling efficiency and sampling convergence, Reliability Engineering \& System Safety, 109: 123-132.

doi: $10.1016 /$ j.ress.2012.08.003

tions: it should be kept in mind that they actually represent a range of values, generally spanning one order of magnitude.

The graphs in Figure 4 confirm that the accuracy of Monte Carlo results increases with increasing numbers of runs, hence falsifying the '100-run barrier' as put forward by Lomas and Eppel [23] and also MacDonald [77]. They suggested that the accuracy does not improve much once above 100 runs. Equations (2) and (3) on the other hand indicate that the accuracy is problem-dependent and Figures 3 and 4 demonstrate a continued decrease of the deviations with increasing numbers of runs. Their '100-run barrier' is hence brought about by their visualisation choices, and should not be taken as a correct guideline.

\subsection{LHS, DIS, UNI sampling}

The LHS sampling efficiency is evaluated similarly as BRS. For the sampling efficiency of DIS and UNI, only 10, 20, 40,60, 80 and 100 runs are investigated as the deviations at 100 runs are closing in on the accuracy limit of the reference solution (Table 1). And as DIS and UNI are quasi-Monte-Carlo approaches - not involving actual random numbers - the generation of the ten repetitions is modified as well. Instead of regenerating any random numbers, ten random permutations of the four columns of the original space-filling design are used. That also implies that executing three series is not meaningful, as this many permutations of four columns are not available: therefore just a single series is simulated for DIS and UNI.

The results are shown in Figures 5 (raw $\mu$ and $\sigma$ values for every n-run set) and Figure 6 (normalised root-mean-square deviations over the ten n-run sets of every series). Figures 5 and 6 show that LHS substantially reduces the deviation from the reference solutions, in comparison to the BRS results. The non-collapsing sampling hence yields a significantly enhanced sampling efficiency. Analogous conclusions are attained for DIS and UNI: they both give an increased accuracy, relative to BRS and LHS. Space-filling sampling (see Figure 1) thus similarly improves the sampling efficiency.

\subsection{Discussion}

Figure 6 clearly depicts the improved sampling efficiency of non-collapsing Latin hypercube sampling over basic random sampling, and of space-filling distance- and uniformity-based designs over standard Latin hypercube designs. Relative to BRS, LHS needs on average 10 times less sampling points for the same accuracy of the estimated $\mu$ and $\sigma$. DIS and UNI further improve the sampling efficiency with respectively a factor 7 and 10, in comparison to standard LHS. Contrary to the conclusion of Macdonald [77] thus, more optimal sampling strategies can be used to reduce the number of required simulation runs in sampling-based uncertainty analyses, and resultantly lessen the computational cost of Monte-Carlo analyses. The superior efficiency of LHS over BRS has been confirmed a number of times already, the superior performance of DIS \& UNI over BRS \& LHS, on the other hand, has not been empirically studied yet to the knowledge of the author. It is established here that application of space-filling Latin hypercube sampling designs strongly improves the sampling efficiency over standard Latin hypercubes.

Based on the work of Niederreiter [78], Fang and co-authors [35] tackle the issue from the 'discrepancy' point of view, leading to interesting conclusions. Quantifying of $\mu_{H L / M G}$ essentially involves the integration of $\mathrm{HL} / \mathrm{MG}$ (equation 1) over the four-dimensional four input parameters - unit hypercube, which in our case is approximated with Monte Carlo. The Koksma-Hlawka inequality gives an upper limit for the approximation error:

$$
\left|I(f)-I^{*}(f, P)\right| \leq V(f) D(P)
$$

where $I$ and $I^{*}$ are the actual and approximated integrals, $f$ an integrand (HL or MG), V(f) the Hardy-Krause variation of $f, P$ the sampling points' set, and $D(P)$ the discrepancy of that set. More precise integration is hence obtained with sampling designs with a lower 
Postprint: Janssen H. 2013. Monte-Carlo based uncertainty analysis: Sampling efficiency and sampling convergence, Reliability Engineering \& System Safety, 109: 123-132.

doi: $10.1016 /$ j.ress.2012.08.003

discrepancy, or in other words, low-discrepancy sampling strategies are more efficient. Fang and co-authors [35] first demonstrate that this discrepancy is, in general, lower for LHS than for BRS, confirming the superior performance of LHS. Their study furthermore confirms that uniform designs have even lower discrepancies, supporting their higher sampling efficiency. The same arguments are true for maximin designs, which also have lower discrepancies than standard Latin hypercube designs.

A concluding illustration of the relative sampling efficiency is brought in Figure 7 , which presents cumulated probability distributions for the heat loss for 10 repetitions of a 100run Monte-Carlo analysis. It is easily noted that space-filling designs like DIS and UNI perform much more robustly than BRS and LHS. Our results hence corroborate crucial advances in the sampling efficiency, and huge reductions in computational expense.

\section{SAMPLING CONVERGENCE}

While efficient sampling strategies may allow reaching a target accuracy with a minimal number of Monte-Carlo runs, the ability to halt the simulations when sufficient accuracy has been attained equally permits reducing the computational expense of Monte Carlo. An appropriate assessment of the sampling convergence and the possibility to sequentially add sampling runs are thus also important. This section introduces a general-purpose method to those aims: a 'sample-splitting bootstrap' approach in combination with 'replicated Latin hypercube' designs. This will be developed stepwise below.

\subsection{Internal measure}

Earlier, in section 5, the accuracy of the Monte Carlo outcomes was evaluated by comparison with the reference solution. Since such reference solution is usually not available, an internal measure of accuracy is preferred. A first step toward this internal measure is replacing the root-mean-square deviation from the series of ten $n$-run analyses from a reference solution with the standard deviation within that series. These normalised internal standard deviations are shown for all computed series in Figure 8. Clearly they compare very well with the original external deviations of Figure 6, which demonstrates there is (virtually) no bias on the $\mu$ and $\sigma$ estimates of BRS, LHS, DIS and UNI.

\subsection{Random sampling}

The previous section corroborated that internal standard deviations on $\mu$ and $\sigma$ can reliably quantify the accuracy of Monte-Carlo results. Their calculation made use of multiple n-run Monte-Carlo sets however, which is computationally expensive. The internal standard deviations can though also be estimated from a single n-run set, from statistical theory, by use of equation (2). By inserting the $\sigma$ and $k$ of the distribution of the targeted outcome, the resultant $\operatorname{std}(\mu)$ and $\operatorname{std}(\sigma)$ are internal single-n-run-set based deviations, indicating the overall accuracy of $\mu$ and $\sigma$. These internal single-n-run-set deviations are compared to the original external 10-n-run-sets deviations in Figure 9: the figure shows that both measures agree well from a global point of view. This technique is therefore implemented in many commercial packages [58]-[60]. Figure 9 however also shows that the internal standard deviations no longer indicate the actual deviations from the reference solutions: instead they should be considered as an indicator of the order of magnitude for the accuracy of n-run Monte Carlo results. Standard bootstrapping can also be used to attain these internal single-n-run-set based deviations: it has been shown previously that this approach gives similar results.

A BRS Monte Carlo analysis can thus be continued by sequentially adding single Monte Carlo runs, until the resulting deviations on $\mu$ and $\sigma$ fall below the desired level. The next paragraph will indicate though that such strategy is not usable for Latin hypercube designs. An alternative, 'reduced bootstrapping without replacement' or 'sample-splitting' [63],[64], is verified here first for random sampling, and is later applied for the Latin 
Postprint: Janssen H. 2013. Monte-Carlo based uncertainty analysis: Sampling efficiency and sampling convergence, Reliability Engineering \& System Safety, 109: 123-132.

doi: $10.1016 /$ j.ress.2012.08.003

hypercube sampling strategies. After $\mathrm{n}$ runs, the $\mathrm{n}$ outcomes are randomly split into $\mathrm{m}$ groups. Averages and standard deviations are calculated for every group, which then yield standard deviations on these averages and standard deviations. The internal single-n-run-set based accuracy indicators for $\mu$ and $\sigma$ are then [39][63],[64],[79]:

$$
\begin{aligned}
& \operatorname{std}(\mu)=\operatorname{std}_{m}\left(\operatorname{avg}_{1-m}\right) / \sqrt{m} \\
& \operatorname{std}(\sigma)=\operatorname{std}_{m}\left(\operatorname{std}_{1-m}\right) / \sqrt{m}
\end{aligned}
$$

where $n$ is the number of runs, $m$ the number of sample-splitting groups, $\mu$ the average of a n-run solution, $\sigma$ the standard deviation of a $n$-run solution, $a v g_{1-m}$ the averages for the $\mathrm{m}$ sample-splitting groups, $s t_{1-m}$ the standard deviations for the $m$ sample-splitting groups, $s_{m}$ the standard deviation of these $m$ averages and standard deviations, and std the expected internal standard deviation.

The predicted std's and their root-mean-squares are also collected in Figure 9. For the study here, only five groups are applied for sample-splitting, while higher numbers are advocated in literature [63],[64]. It can be seen though that such low number of sample-splitting groups gives results very similar to the sampling statistics, and that they moreover compare well with the original external deviations, at least in reference to their global order of magnitude.

\subsection{LHS, DIS, UNI sampling}

Unlike random sampling based Monte Carlo, Latin hypercube sampling schemes do not easily allow sequentially adding simulation runs till the desired accuracy is reached: the number of runs is usually determined before starting the Monte Carlo analysis (although multi-level or sequential Latin hypercube designs are being introduced [65],[66]) and that translates to the number of equiprobable strata applied. Only when all planned samples have been run is the representative sampling of the separate parameter spaces, and of the multi-parameter space, obtained. Any intermediate result is therefore not necessarily better than its related BRS result (at the same number of runs). Assessing the sampling convergence of standard Latin hypercube sampling results is equally trying: while Latin hypercube is a variance-reduction technique, no continuous quantification of how much the variance actually is is available. Standard bootstrapping cannot be used, as this equally ignores the specific sample selection, and the sampling statistics' equation (2) is neither valid for Latin hypercube sampling designs.

Assessment of the sampling convergence and sequential addition of sampling runs can however be made possible by use of replicated Latin hypercubes, as first introduced by Iman [39] and further developed by McKay [79]. Instead of the single n-run Latin hypercube design, McKay has suggested to use permutated repetitions of smaller designs to reach the set number of runs $n$. Such replicated designs hence allow adding additional runs to a Monte Carlo analysis, albeit in blocks of runs instead of single runs. The replicated designs moreover allow monitoring the convergence of the Monte Carlo results, since they can be used as the sample-splitting groups in the suggested reduced bootstrapping approach. The combination of replicated Latin hypercube designs and sample-splitting bootstrapping hence permits halting a Latin hypercube Monte Carlo analysis when the desired level of accuracy has been reached.

Exemplarily, it is developed here based on the 100-run UNI set. Instead of the original 100 -sample uniform sampling design, five random variations (column permutations) of the 20-point uniform design are used to obtain the 100 desired samples. The resulting normalised (root-mean-square) external deviations (deviation from the reference solution) of this replicated approach are compared with the original n-run deviations in Figure 10. It is obvious that the replicated uniform designs lead to similar performances as the original designs. An additional improvement can be obtained by splitting a 100-run UNI 
Postprint: Janssen H. 2013. Monte-Carlo based uncertainty analysis: Sampling efficiency and sampling convergence, Reliability Engineering \& System Safety, 109: 123-132.

doi: $10.1016 /$ j.ress.2012.08.003

design into a number of smaller subdesigns, each with an optimal uniformity within those constraints [12], but for the development here the pure replication approach is assumed sufficient.

The replicated approach in turn consequentially also allows application of sample-splitting, based on the different 20-run sets, via equation (5), to reach the desired accuracy estimates. The normalised internal standard deviations, as predicted with sample-splitting, are also shown in Figure 10. For the values at 100 runs five resampling groups of 20 runs each are applied, at 80 runs four resampling groups, ... Minimally two samplesplitting groups are needed, so 40 runs is the lower limit for the replicated 20 approach. The comparison with the original external deviations for the replicated 20 indicates that sample-splitting reliably predicts the accuracy of the obtained $\mu$ and $\sigma$. This is also valid for the 40-run results, based on just two resampling groups.

\subsection{Discussion}

The previous section demonstrated that non-collapsing space-filling sampling designs allow obtaining a certain accuracy of the targeted outcomes with far less runs than basic random sampling. Determining the number of runs required to arrive at the desired level of accuracy is a different matter altogether, specially for Latin hypercube sampling designs. In standard applications of these, the number of runs is to be predetermined. Moreover, no easy quantification of the variances on the targeted outcomes is possible, hindering an evaluation of the accuracy of the obtained results.

To resolve both issues, a general-purpose method for gauging the accuracy of Latin hypercube Monte Carlo results has been put forward. It has been shown that root-meansquare deviations from the reference solution can be reliably estimated by the internal standard deviations on the resulting $\mu$ and $\sigma$. It has moreover been shown that the latter can be estimated with a sample-splitting approach. Combination with the replicated Latin hypercube sampling designs (illustrated here for UNI but equally applicable to DIS and LHS) allows evaluating the variances on the Monte Carlo outcomes, which in turn permits halting the calculation when the desired accuracy levels are reached. It should be kept in mind however that, as mentioned before, that the internal measures of accuracy only provide an order of magnitude, and not a sharp result.

This is illustrated in Figure 11 (left), confronting the external deviation - the actual deviation from the reference solution - with the internal deviation - the estimate of the deviation based on sample-splitting - for every distinct n-run set performed in the BRS and replicated UNI investigation. It can be seen that a fair agreement exists, but a serious amount of noise is present as well. The same is true though when comparing external deviations with internal deviations from equation (2), this time only for the BRS sets (Figure 11, right). The introduced sample-splitting approach for quantification of the accuracy thus gives a reliability similar to that of sampling statistics, but is far more generally applicable. And while the developments here have focused on the mean and the standard deviation, these can easily be replaced by other measures as well.

\section{Closing example}

To yet again show the merits of improving sampling efficiency and monitoring sampling convergence, a probabilistic design of the natural ventilation for a single-family house is executed here. To prevent excessive interior humidity or poor interior air quality, the air change rates of residential buildings should remain above a certain lower limit; similarly a certain upper limit is desired to avoid undue energy consumption or possible draught complaints. Since the uncertainty on the driving forces for natural ventilation - thermal stack and wind pressure - is fairly significant, a probabilistic design method is required to obtain a reliable ventilation solution. 
Postprint: Janssen H. 2013. Monte-Carlo based uncertainty analysis: Sampling efficiency and sampling convergence, Reliability Engineering \& System Safety, 109: 123-132.

doi: $10.1016 /$ j.ress.2012.08.003

The calculation object used here is a case introduced in [80][81]: natural ventilation of a test house in Sweden. Based on the characteristics of test house and building location, the following expression for the air change rate caused by thermal stack and wind pressures was developed:

$$
\begin{array}{rlrl}
A C H & =A_{\text {corr }} \sqrt{\mathrm{s}_{1}|\Delta T|^{2}+\mathrm{s}_{2}|\Delta \mathrm{T}|+\mathrm{s}_{3}|\Delta \mathrm{T}|^{1.5}+\mathrm{w}_{1} \mathrm{v}^{4}+\mathrm{w}_{2} \mathrm{v}^{2}+\mathrm{w}_{3} \mathrm{v}^{3}} \\
\mathrm{~s}_{1} & =1.3810^{-3} 1 / \mathrm{h}^{2} \mathrm{~K}^{2} & \mathrm{w}_{1}=4.9510^{-5} \mathrm{~s}^{4} / \mathrm{h}^{2} \mathrm{~m}^{4} \\
\mathrm{~s}_{2} & =3.3810^{-1} 1 / \mathrm{h}^{2} \mathrm{~K} & \mathrm{w}_{2}=5.4610^{-3} \mathrm{~s}^{2} / \mathrm{h}^{2} \mathrm{~m}^{2} \\
\mathrm{~s}_{3} & =4.3110^{-2} 1 / \mathrm{h}^{2} \mathrm{~K}^{1.5} & \mathrm{w}_{3}=1.0410^{-3} \mathrm{~s}^{3} / \mathrm{h}^{2} \mathrm{~m}^{3}
\end{array}
$$

where $\mathrm{ACH}[1 / \mathrm{h}]$ is the air change rate of the house, $\mathrm{A}_{\text {corr }}[-]$ the ratio of the required and current areas of leakage openings, $\Delta \mathrm{T}[\mathrm{K}]$ the interior-exterior temperature difference, $\mathrm{v}$ $[\mathrm{m} / \mathrm{s}]$ the wind speed at the site of the house, and $\mathrm{s}_{\mathrm{i}}$ and $\mathrm{w}_{\mathrm{i}}$ are coefficients. The values for $w_{i}$ given here relate only to winds coming from the South, and for reasons of brevity the analysis is restricted to that wind direction only. Extension to the complete range of wind directions is straightforward though.

The uncertain parameters for this example are the temperature difference and the wind speed, distributions of which were drawn from 40-year climate sets [80]: a normal distribution for $\Delta \mathrm{T}$ with mean $10.2 \mathrm{~K}$ and standard deviation $3.8 \mathrm{~K}$, a Weibull distribution for $\mathrm{v}$ with scale parameter $2.51 \mathrm{~m} / \mathrm{s}$ and shape parameter 2.33. The correlation between the two parameters was moreover shown to be negligible. The lower and upper limit for the air change rate of are set here at $0.4 / \mathrm{h}$ and $1.0 / \mathrm{h}$. While these specific values can certainly be debated, they are sufficiently reasonable for the example at hand. The specific design objective is the most reliable ventilation, by minimising the probability of an $\mathrm{ACH}$ outside of the set range, through modifications of the design factor $A_{c o r r}$. The probability of unfit ventilation is quantified via Monte Carlo, using the "replicated 20" approach with UNI sampling designs, and with the convergence limit set at a relative error of $5 \%$. For better stability that probability is derived analytically, via a lognormal distribution fitted to the Monte Carlo outcomes [80], instead of using the simple hits outside the $0.4 / \mathrm{h}$ and 1 $/ h$ interval.

The converged Monte Carlo outcomes for $\mathrm{ACH}$, shown as the fitted lognormal distributions and probabilities of unfit ventilation, are collected in Figure 12 (left) for $A_{\text {corr }} 1.5,2.1$, and 2.7. The most reliable ventilation solution is found for $A_{\text {corr }}$ at 2.1, with an unfit ventilation probability of about $8.5 \%$. For this specific solution, the converged Monte Carlo outcome results from 40 runs, at which the relative error is already just below $4 \%$. The same analysis is finally performed with BRS sampling designs, attaining a similar result but necessitating 3100 runs to reach the $5 \%$ convergence criterion. The convergence of the UNI and BRS Monte Carlo analyses is illustrated in Figure 12 (right): the UNI-based analysis gets down to $1 \%$ relative error at 200 runs, while the BRS-based analysis requires several tens of thousand runs to attain the same level of convergence. These observations once more illustrate the benefits of the developments in this paper: 'monitoring sampling convergence' permits the cessation of Monte Carlo analyses when sufficient accuracy is reached, 'improving sampling efficiency' allows the attainment of that accuracy with a minimal number of Monte Carlo runs.

\section{CONCLUSIONS}

Monte Carlo analysis has become nearly ubiquitous since its introduction, now over 65 years ago. It is an important tool in many assessments of the reliability and robustness of systems, structures or solutions. That is equally true for the stochastic simulation of heat and moisture transfer in whole buildings, building components and building materials, where the application of Monte-Carlo-based uncertainty analysis currently prevails. As the deterministic core simulation may at times be very lengthy however, the compu- 
Postprint: Janssen H. 2013. Monte-Carlo based uncertainty analysis: Sampling efficiency and sampling convergence, Reliability Engineering \& System Safety, 109: 123-132.

doi: $10.1016 /$ j.ress.2012.08.003

tational costs of Monte Carlo can be a limiting factor. To reduce that computational expense as much as possible hence, this paper has studied sampling efficiency and convergence for Monte Carlo.

In a first section it was shown that non-collapsing space-filling sampling designs, exemplified here with maximin and uniform designs, highly improve sampling efficiency, rendering the desired level of accuracy of the targeted outcomes attainable with far lesser runs. Use of space-filling Latin hypercube designs is hence generally recommendable. In a second section it was demonstrated that sampling statistics or standard bootstrapping are not applicable for Latin hypercube sampling strategies. Instead, a replicationbased Latin hypercube sampling was proposed, giving similar performance as the related original design. This replication strategy was combined with a sample-splitting approach, allowing quantification of the accuracy of the Monte Carlo results and thus permitting to halt the analysis when sufficient accuracy is obtained.

Both measures form fairly noncomplex upgrades of the current state-of-the-art in Monte-Carlo-based uncertainty analysis, but lead to an important further progress with respect to its applicability.

\section{ACKNOWLEDGEMENT}

Thank you to Bart Husslage and Gijs Rennen from the Tilburg School of Economics and Management, Tilburg University, the Netherlands, for sharing the Matlab code for development of maximin Latin hypercube designs [82].

\section{REFERENCES}

[1] Helton JC, Burmaster DE. Guest editorial: treatment of aleatory and epistemic uncertainty in performance assessments for complex systems. Reliab Eng Syst Safe 1996;54:91-4.

[2] Oberkampf WL, DeLand SM, Rutherford RM, Diegert KV, Alvin KF. Error and uncertainty in modeling and simulation. Reliab Eng Syst Safe 2002; 75:333-57.

[3] Helton JC. Treatment of uncertainty in performance assessments of complex system. Risk Anal 2004;14:483-511.

[4] Karadeniz H, Vrouwenvelder T. Overview reliability methods. In: Safety and Reliability of Industrial Products, Systems and Structures, Guedes Soares, C., (Ed.), Taylor \& Francis Group, London, United Kingdom, 2010, pp. 181-9.

[5] Burmaster DE, Anderson PD. Principles of good practice for the use of monte carlo techniques in human health and ecological risk assessments. Risk Anal 1994;14:477-81.

[6] Ferson S. What Monte Carlo methods cannot do. Hum Ecol Risk Assesss 1996; 2:990-1007.

[7] Moore DRJ. Using Monte Carlo analysis to quantify uncertainty in ecological risk assessment: are we gilding the lily or bronzing the dandelion ? Hum Ecol Risk Assesss 1996;2:628-33.

[8] Singhee A, Rutenbar RA. Why quasi-Monte Carlo is better than Monte Carlo or Latin hypercube Sampling for statistical circuit analysis. IEEE Trans. ComputerAided Design Integr Circuits Syst. 2010;29:1763-76.

[9] Cardoso JB, de Almeida JR, Diasa JM, Coelho PG. Structural reliability analysis using Monte Carlo simulation and neural networks. Adv Eng Softw 2008;39: 505-13.

[10] Helton JC, Hansen CW, Sallaberry CJ. Uncertainty and sensitivity analysis in performance assessment for the proposed repository for high-level radioactive waste at Yucca Mountain, Nevada. Reliab Eng Syst Safe; In press.

[11] Rugen P, Callahan B. An overview of Monte Carlo - a fifty year perspective. Hum Ecol Risk Assesss 1996;2:671-80. 
Postprint: Janssen H. 2013. Monte-Carlo based uncertainty analysis: Sampling efficiency and sampling convergence, Reliability Engineering \& System Safety, 109: 123-132.

doi: $10.1016 /$ j.ress.2012.08.003

[12] Dehlendorff C, Kulahci M, Andersen KK. Designing simulation experiments with controllable and uncontrollable factors for applications in healthcare. J Roy Stat Soc C-App 2011;60:31-49.

[13] Van Groenendaal WJH, Kleijnen JPC. On the assessment of economic risk: factorial design versus Monte Carlo methods. Reliab Eng Syst Safe 1997;57:91 $-102$.

[14] Cioppa TM, Lucas TW. Efficient nearly orthogonal and space-filling Latin hypercubes. Technometrics 2007;49:45-55.

[15] Yin L, Smith MAJ, Trivedi KS. Uncertainty analysis in reliability modeling, in: Proceedings of Annual Reliability \& Maintainability Symposium, Philadelphia, Pennsylvania, USA, 2001.

[16] Durga Rao K, Gopika V, Sanyasi Rao VVS, Kushwaha HS, Verma AK, Srividya A. Dynamic fault tree analysis using Monte Carlo simulation in probabilistic safety assessment. Reliab Eng Syst Safe 2009;94:872-883.

[17] Hammersley JM. Monte Carlo methods for solving multivariable problems. Ann NY Acad Sci 1960;86:844-74.

[18] Li Z, Chen W, Deng S, Lin Z. The characteristics of space cooling load and indoor humidity control for residences in the subtropics. Build Environ 2006;41: 1137-47.

[19] Pasanen A-L, Kasanen J-K, Rautiala S, Ikäheimo M, Rantamäki J, Kääriäinen H et al. Fungal growth and survival in building materials under fluctuating moisture and temperature conditions. Int Biodeter Biodegr 2000;46:117-27.

[20] Mudarri D, Fisk WJ. Public health and economic impact of dampness and mold. Indoor Air 2007;17:226-35.

[21] Fang L, Clausen G, Fanger PO. Impact of temperature and humidity on the perception of indoor air quality. Indoor Air 1998;8:80-90.

[22] Hokoi S, Matsumoto M. An analysis of stochastic properties of the heating load in an intermittently air-conditioned building. Energ Buildings 1988; 11:259-66.

[23] Lomas KJ, Eppel H. Sensitivity analysis techniques for building thermal simulation programs. Energ Buildings 1992;19:21-44.

[24] Macdonald IA, Clarke JA. Applying uncertainty considerations to energy conservation equations. Energ Buildings 2007;39:1019-26.

[25] de Wit S, Augenbroe G. Analysis of uncertainty in building design evaluations and its implications. Energ Buildings 2002;34:951-58.

[26] Pietrzyk K, Kurkinen K, Hagentoft C-E. An example of application of limit state approach for reliability analysis of moisture performance of a building component. J. Therm. Envelope Build. Sci. 2004;28:9-26.

[27] Haarhoff $J$, Mathews EH. A Monte Carlo method for thermal building simulation. Energ Buildings 2006;38:1395-99.

[28] Corrado V, Mechri HE. Uncertainty and sensitivity analysis for building energy rating. Journal of Building Physics 2009;33:125-156.

[29] Yun GY, Tuohy P, Steemers K. Thermal performance of a naturally ventilated building using a combined algorithm of probabilistic occupant behaviour and deterministic heat and mass balance models. Energ Buildings 2009;41:489-99.

[30] Domínguez-Muñoz F, Cejudo-López JM, Carrillo-Andrés A. Uncertainty in peak cooling load calculations. Energ Buildings 2010;42:1010-18.

[31] Xu C, He HS, Hu Y, Chang Y, Li X, Bu R. Latin hypercube sampling and geostatistical modeling of spatial uncertainty in a spatially explicit forest landscape model simulation. Ecol Model 2005;185:255-69.

[32] McKay MD, Beckman RJ, Conover WJ. A comparison of three methods for selecting values for input variables in the analysis of output from a computer code. Technometrics 1979;21:239-45. 
Postprint: Janssen H. 2013. Monte-Carlo based uncertainty analysis: Sampling efficiency and sampling convergence, Reliability Engineering \& System Safety, 109: 123-132.

[33] Johnson M.E., Moore L.M., Ylvisaker D. Minimax and maximin distance designs. J Stat Plan Infer 1990;26:131-48.

[34] Morris MD, Mitchell TJ. Exploratory designs for computational experiments. J Stat Plan Infer 1995;43:381-402.

[35] Fang K-T, Ma C-X, Winker P. Centered L2-discrepancy of random sampling and Latin hypercube design, and construction of uniform designs. Math Comput 200;71:275-96.

[36] Joseph VR, Hung Y. Orthogonal-maximin Latin hypercube designs. Stat Sinica 2008;18:171-86.

[37] Pronzato L, Müller WG. Design of computer experiments: space filling and beyond. Stat Comput 2011; online first.

[38] Gilman MJ. A brief survey of stopping rules for Monte Carlo. in: Proceedings of the Second Conference on Applications of Simulations, New York, New York, USA, 1968

[39] Iman RL. Statistical methods for including uncertainties associated with the geologic isolation of radioactive waste which allow for comparison with licensing criteria. In: Proceedings of the Symposium on Uncertainties Associated with the Regulation of the Geologic Disposal of High-Level Radioactive Waste, Gatlinburg, Tennessee, USA, 1981.

[40] Hansen CW, Helton CJ, Sallaberry CJ. Use of replicated Latin hypercube sampling to estimate sampling variance in uncertainty and sensitivity analysis results for the geologic disposal of radioactive waste. Reliab Eng Syst Safe, in press.

[41] Bielajew AF. Fundamentals of the Monte Carlo method for neutral and charged particle transport. The University of Michigan, Ann Arbor, Michigan, USA, 2001.

[42] Walters BRB, Kawrakow I, Rogers DWO. History by history statistical estimators in the BEAM code system. Med Phys 2002;29:2745-52.

[43] Stein M. Large sample properties of simulations using Latin hypercube sampling. Technometrics 1987;29:143-51.

[44] Helton JC, Davis FJ. Latin hypercube sampling and the propagation of uncertainty in analyses of complex systems, SAND2001-0417, Sandia National Laboratories, New Mexico, USA, 2002.

[45] Helton JC, Davis FJ. Latin hypercube sampling and the propagation of uncertainty in analyses of complex systems. Reliab Eng Syst Safe 2003;81:23-69.

[46] Helton JC, Davis FJ, Johnson JD. A comparison of uncertainty and sensitivity analysis results obtained with random and Latin hypercube sampling. Reliab Eng Syst Safe 2005;89:305-30.

[47] Hossain F, Anagnostou EN, Bagtzoglou AC. On Latin Hypercube sampling for efficient uncertainty estimation of satellite rainfall observations in flood prediction. Comput Geosci 2006;32:776-92.

[48] Khan AA, Lye L, Husain T. Latin hypercube sampling for uncertainty analysis in multiphase modeling. J Environ Eng Sci 2008;7:617-26.

[49] Pilger GG, Costa JF, Koppe J. The benefits of Latin Hypercube Sampling in sequential simulation algorithms for geostatistical applications. App Earth Sci 2008;117:160-74.

[50] Santner TJ, Williams BJ, Notz WI. The design and analysis of computer experiments, Springer-Verlag - New York, New York, United States, 2003.

[51] Fang K-T, Li R, Sudjianto A. Design and modelling for computer experiments, Taylor \& Francis Group, Boca Raton, Florida, United States, 2006.

[52] Simpson TW, Lin TKJ, Chen W. Sampling strategies for computer experiments: design and analysis. Int J Reliab App 2001;2:209-40. 
Postprint: Janssen H. 2013. Monte-Carlo based uncertainty analysis: Sampling efficiency and sampling convergence, Reliability Engineering \& System Safety, 109: 123-132.

[53] Giunta AA, Wojtkiewicz Jr. SF, Eldred MS. Overview of modern design of experiments methods for computational simulations, in: Proceedings of $41^{\text {st }}$ AIAA Aerospace Sciences Meeting and Exhibit, Reno, Nevada, USA, 2003.

[54] Chen VCP, Tsui K-L, Barton RR, Allen JK. A review of design and modeling in computer experiments, in Handbook of Statistics, 2003, Volume 22, 231-61.

[55] Johnson RT, Montgomery DC, Bradley J, Parker PA. Comparing computer experiments for fitting high-order polynomial metamodels. J Qual Technol 2010;42 :86-102.

[56] Alam FM, McNaught KR, Ringrose TJ. A comparison of experimental designs in the development of a neural network simulation metamodel. Simulat Model Pract Theor 2004;12:559-78.

[57] Simlab - Software package for uncertainty and sensitivity analysis. Joint Research Centre of the European Commission. Downloadable for free at: simlab.jrc.ec.europa.eu, last accessed November 42011.

[58] Palisade Corporation. Guide to using @RISK, Risk Analysis and Simulation Add-In for Microsoft巴 Excel, Version 5.7, Ithaca, New York, USA, 2010.

[59] Frontline Systems. Frontline Solvers User Guide, Incline Village, Nevada, USA, 2011.

[60] Oracle Crystal ball. www.oracle.com/technetwork/middleware/crystalball/overview/precision-control-133236.pdf, last accessed on November 42011.

[61] Efron B, Tibshirani R. Bootstrap methods for standard errors, confidence intervals, and other measures of statistical accuracy. Stat Sci 1986;1:54-75.

[62] Booth JG, Sarkar S. Monte Carlo approximation of bootstrap variances. Am Stat 1998;52:354-57.

[63] Bickel PJ, Götze F, van Zwet WR. Resampling fewer than n observations: gains, losses, and remedies for losses. Stat Sinica 1997;7:1-31.

[64] Pino Mej R, Jimenez Gamero MD, Enguix Gonzalez A. A Monte Carlo comparison of three consistent bootstrap procedures. J Stat Comput Simul 2009;79: 323-34.

[65] Sallaberry CJ, Helton JC, Hora SC. Extension of Latin Hypercube samples with correlated variables. Reliab Eng Syst Safe 2008;93:1047-59.

[66] Xiong F. Optimizing Latin hypercube design for sequential sampling of computer experiments. Eng Optim 2009;41:793-810.

[67] Hagentoft C-E, Kalagasidis AS, Adl-Zarrabi B, Roels S, Carmeliet J, Hens H et al. Assessment method of numerical prediction models for combined heat, air and moisture transfer in building components: benchmarks for one-dimensional cases. J Therm Envelope Build Sci 2004;27:327-352.

[68] Hagentoft C-E. HAMSTAD WP2 Benchmarking package, final report. Document Chalmers2002-h14, Chalmers University of Technology, Göteborg, Sweden, 2002.

[69] Janssen $\mathrm{H}$, Blocken B, Carmeliet J. Conservative modelling of the moisture and heat transfer in building components under atmospheric excitation. Int $\mathrm{J}$ Heat Mass Transfer 2007;50:1128-40.

[70] Janssen $\mathrm{H}$. Adaptive Kronrod-Patterson integration of non-linear finite-element matrices. Int J Numer Methods Eng 2010;81:1455-74.

[71] Janssen $\mathrm{H}$. Thermal diffusion of water vapour in porous materials: fact or fiction ? Int J Heat Mass Transfer 2011;54:1548-62.

[72] Janssen $\mathrm{H}$, Blocken $\mathrm{B}$, Roels $\mathrm{S}$, Carmeliet $\mathrm{J}$. Wind-driven rain as a boundary condition for HAM simulations: analysis of simplified modelling approaches. Build Environ 2007;42:1555-67.

[73] Iman RL, Conover WJ. A Distribution-Free Approach to Inducing Rank Correlation Among Input Variables. Commun Stat Simul Comput 1982; B11:311-334. 
Postprint: Janssen H. 2013. Monte-Carlo based uncertainty analysis: Sampling efficiency and sampling convergence, Reliability Engineering \& System Safety, 109: 123-132.

[74] Owen AB. Controlling correlations in Latin hypercube samples. J Amer Stat Assoc 1994;89:1517-22

[75] Jin R, Chen W, Sudjianto A. An efficient algorithm for constructing optimal design of computer experiments. J Stat Plan Infer 2005;134:268-87.

[76] Wikipedia. en.wikipedia.org/wiki/Variance, last accessed on November 42011.

[77] Macdonald IA, 2009. Comparison of sampling techniques on the performance of Monte Carlo based sensitivity analysis, in: Proceedings of Building Simulation 2009, 11th International IBPSA Conference, Glasgow, United Kingdom, 2009.

[78] Niederreiter H. Random number generation and quasi-Monte Carlo methods. Society for industrial and applied mathematics, Philadelphia, Pennsylvania, 1192.

[79] McKay MD. Evaluating prediction uncertainty, Los Alamos National Laboratory, Report NUREG/CR-6311, Los Alamos, New Mexico, USA, 1995.

[80] Pietrzyk K, Hagentoft C-E. Probabilistic analysis of air infiltration in low-rise buildings. Build Environ 2008;43:537-49.

[81] Pietrzyk K, Hagentoft C-E. Reliability analysis in building physics design. Build Environ 2008;43:558-68.

[82] Dam ER van, Hertog D den, Husslage BGM, Rennen G. Space-filling Latin hypercube designs for computer experiments. Optim Eng 2011;12:611-30. 
Postprint: Janssen H. 2013. Monte-Carlo based uncertainty analysis: Sampling efficiency and sampling convergence, Reliability Engineering \& System Safety, 109: 123-132.

\section{TABLES}

Table 1. $\mu$ and $\sigma$ for $\mathrm{HL}$ and MG from the reference solution, with normalised standard deviations on these values.

\begin{tabular}{|c|c|c|c|}
\hline$\mu_{\mathrm{HL}}[\mathrm{MJ}]$ & $\sigma_{\mathrm{HL}}[\mathrm{MJ}]$ & $\mu_{\mathrm{MG}}[\mathrm{kg}]$ & $\sigma_{\mathrm{MG}}[\mathrm{kg}]$ \\
\hline 14.97 & 3.112 & 3.406 & 0.9519 \\
\hline \multicolumn{4}{|c|}{ normalised standard deviations: statistics } \\
\hline $0.07 \%$ & $0.05 \%$ & $0.09 \%$ & $0.11 \%$ \\
\hline \multicolumn{4}{|c|}{ normalised standard deviations: bootstrap } \\
\hline $0.06 \%$ & $0.04 \%$ & $0.08 \%$ & $0.10 \%$ \\
\hline
\end{tabular}

\section{FIGURE CAPTIONS}

FIG 1. Sampling desings for BRS (top left), LHS (top right), DIS (bottom left), UNI (bottom right): full markers are the actual sampling points, hollow markers are their projections onto the two separate dimensions.

FIG 2. Cumulated probability distributions for the $H L$ and MG reference solutions.

FIG 3. Predicted $\mu$ and $\sigma$ for BRS with different number of runs $n$; the black lines indicate the reference solutions (full: $\mu$; dashed: $\sigma$ ).

FIG 4. Normalised (root-mean-square) deviations from the reference solutions, for three series of ten $n$-run BRS sets. Hollow markers depict the normalised deviations, lines show their root-mean-square averages.

FIG 5. LHS (top) and DIS \& UNI (bottom) predicted $\mu$ and $\sigma$ with different number of runs $\mathrm{n}$; the black lines indicate the reference solutions (full: $\mu$; dashed: $\sigma$ ).

FIG 6. Normalised root-mean-square deviations from the reference solutions, for three BRS and LHS series and one DIS and UNI series.

FIG 7. Cumulated probability distributions of $\mathrm{HL}$ for a series of ten 100-run Monte Carlo simulations, in comparison to the reference solution (black curve).

FIG 8. Normalised internal standard deviations for all series of ten n-run Monte-Carlo sets.

FIG 9. Normalised external deviations and normalised internal standard deviations (statistical and sample-split) for one BRS series of ten n-run Monte-Carlo sets. Hollow markers depict the normalised deviations, lines show their root-mean-squares.

FIG 10. Normalised external deviations and normalised internal standard deviations (sample-split) for the original UNI results and the replicated-20 UNI results. Hollow markers depict the normalised deviations, lines show their root-mean-squares.

FIG 11. Comparison of normalised external deviations and normalised internal deviations - based on sample-splitting estimates - for separate n-run sets from the BRS and replicated UNI investigation (left); comparison of normalised external deviations and normalised internal deviations - based on sampling-statistics estimates - for separate n-run sets from the BRS (right).

FIG 12. Resulting ACH distributions for Acorr 1.5, 2.1, 2.7, and the related probabilities of unfit ventilation (left); comparison of the convergence of the Monte Carlo analyses using UNI and BRS designs for the optimal Acorr 2.1 (right). 
Postprint: Janssen H. 2013. Monte-Carlo based uncertainty analysis: Sampling efficiency and sampling convergence, Reliability Engineering \& System Safety, 109: 123-132.
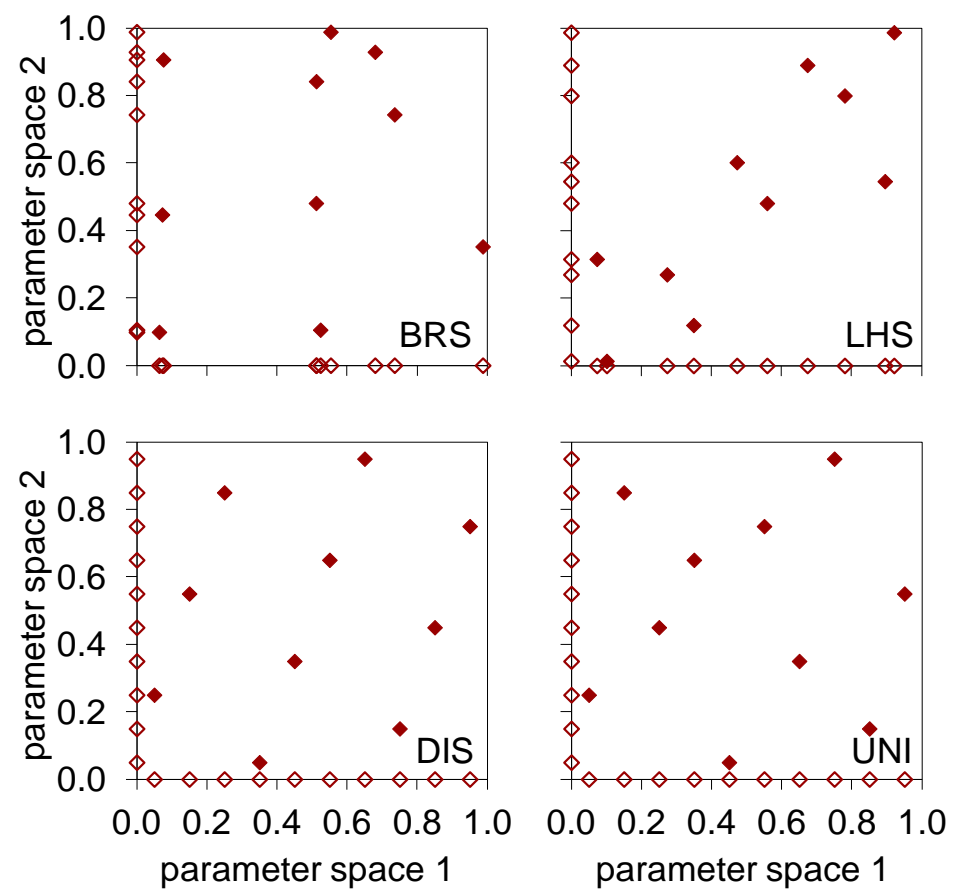

FIG 1. BRS, LHS, DIS, UNI sampling designs: full markers are actual sampling points, hollow markers are projections onto the two separate dimensions.
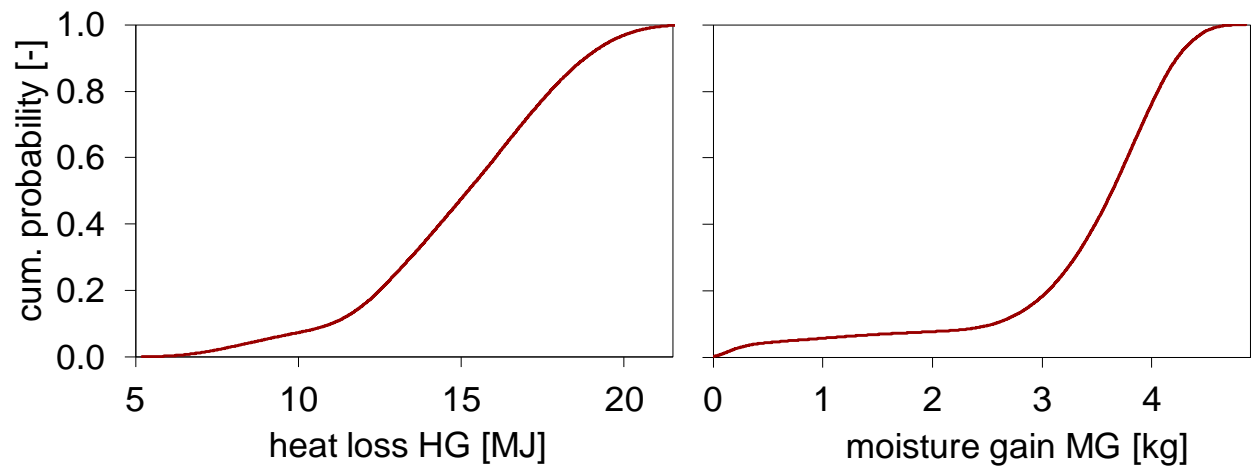

FIG 2. Cumulated probability distributions for the HL and MG reference solutions.
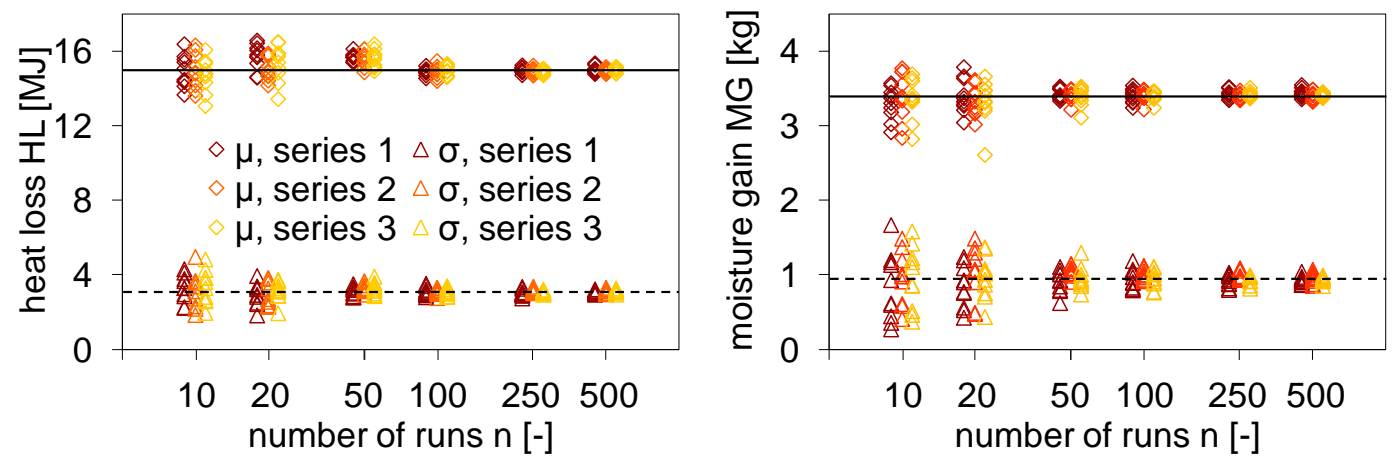

FIG 3. Predicted $\mu$ and $\sigma$ for BRS with different number of runs $n$; the black lines indicate the reference solutions (full: $\mu$; dashed: $\sigma$ ). 
Postprint: Janssen H. 2013. Monte-Carlo based uncertainty analysis: Sampling efficiency and sampling convergence, Reliability Engineering \& System Safety, 109: 123-132.
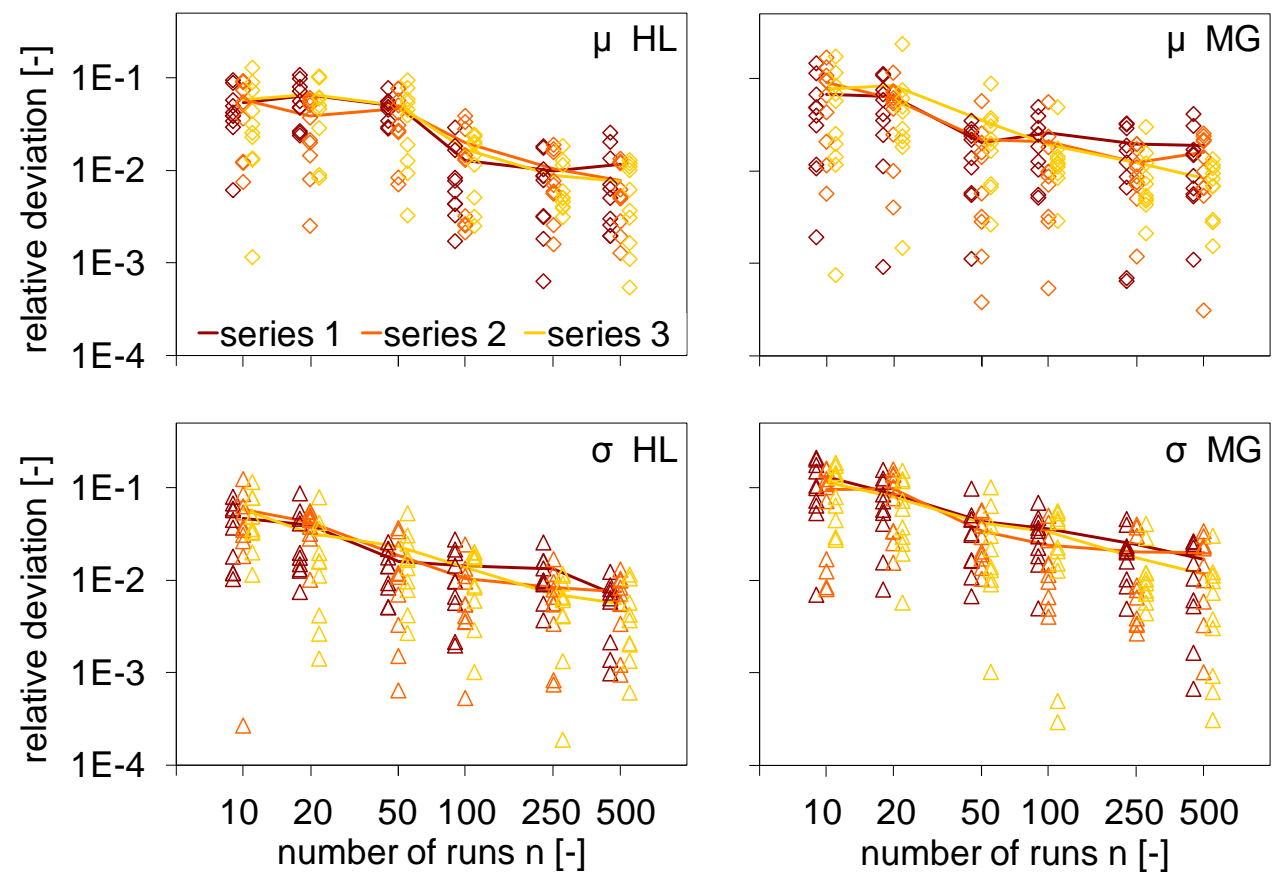

FIG 4. Normalised (root-mean-square) deviations from the reference solutions, for three series of ten $\mathrm{n}$-run BRS sets. Hollow markers depict the normalised deviations, lines show their root-mean-squares.
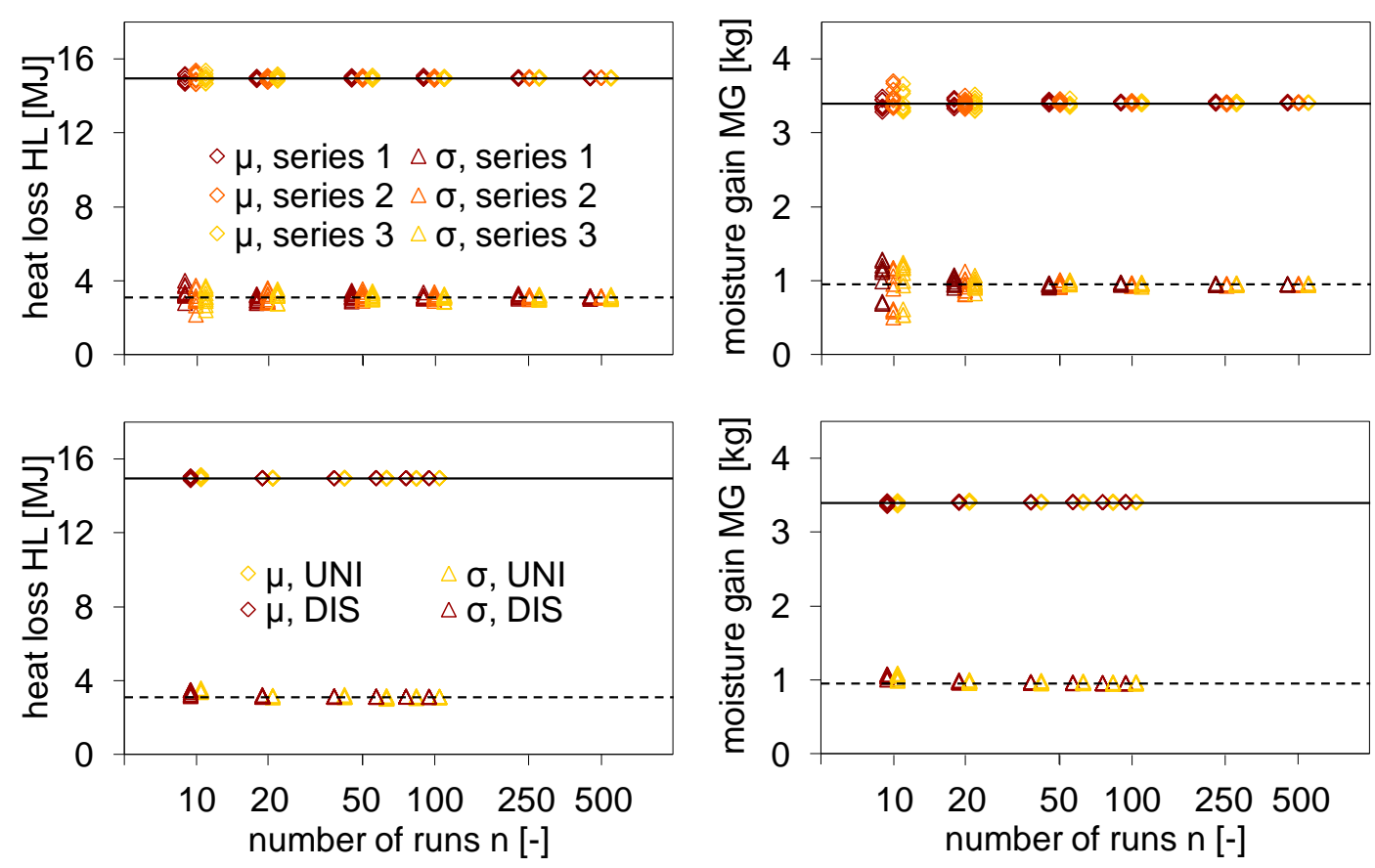

FIG 5. LHS (top) and DIS \& UNI (bottom) predicted $\mu$ and $\sigma$ with different number of runs $\mathrm{n}$; the black lines indicate the reference solutions (full: $\mu$; dashed: $\sigma$ ). 
Postprint: Janssen H. 2013. Monte-Carlo based uncertainty analysis: Sampling efficiency and sampling convergence, Reliability Engineering \& System Safety, 109: 123-132.
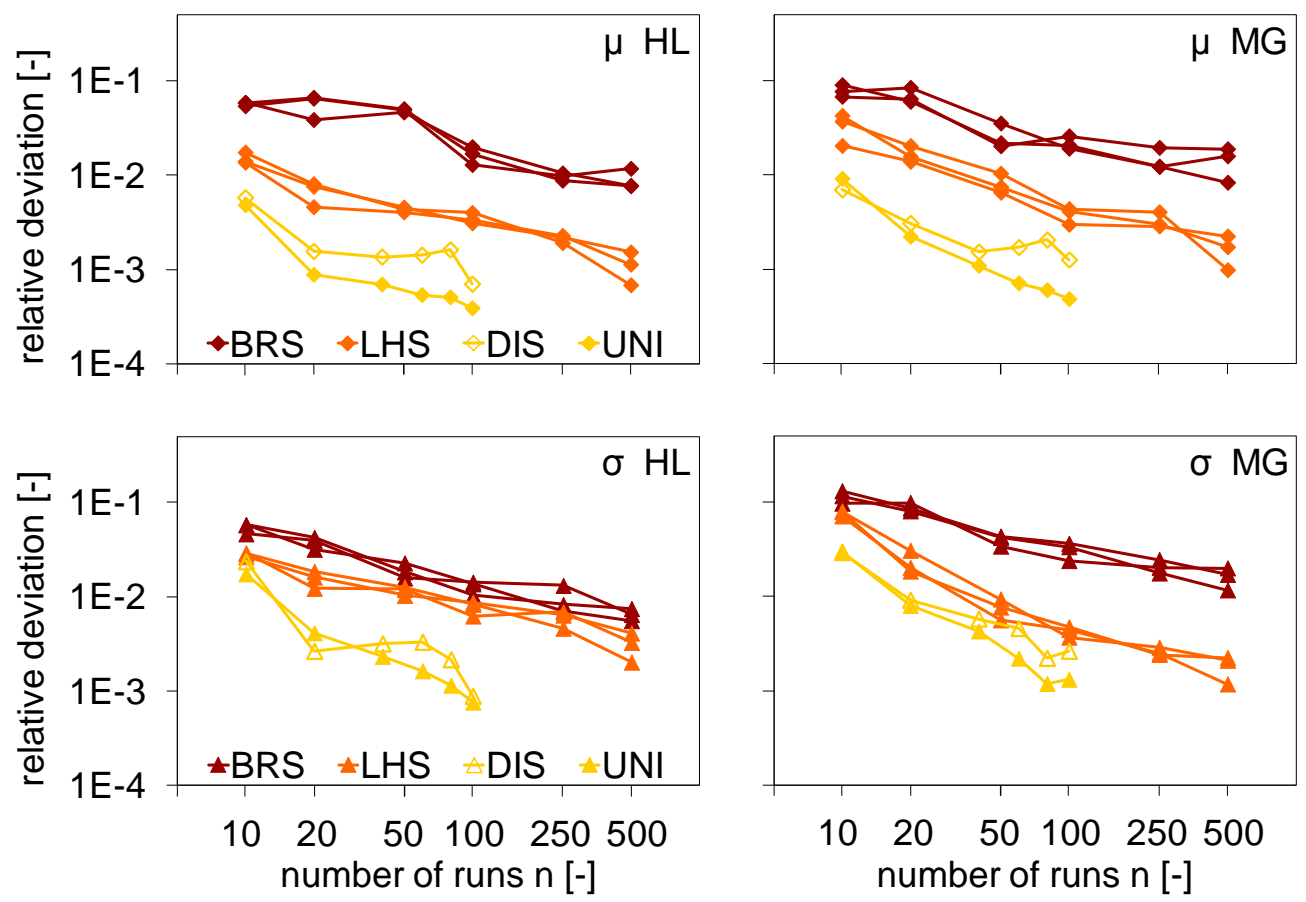

FIG 6. Normalised root-mean-square deviations from the reference solutions, for three BRS and LHS series and one DIS and UNI series.
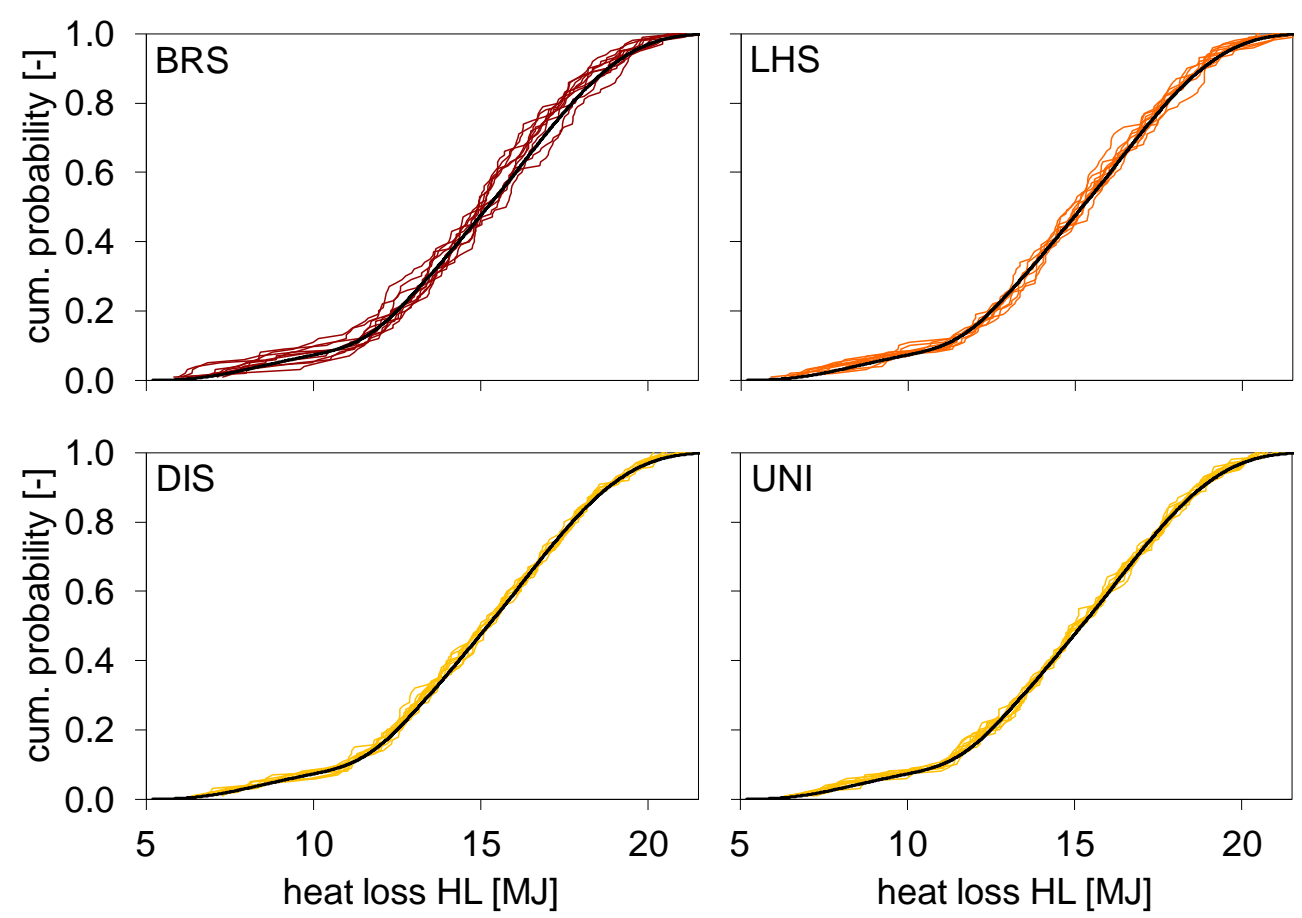

FIG 7. Cumulated probability distributions of $\mathrm{HL}$ for a series of ten 100-run Monte Carlo simulations, in comparison to the reference solution (black curve). 
Postprint: Janssen H. 2013. Monte-Carlo based uncertainty analysis: Sampling efficiency and sampling convergence, Reliability Engineering \& System Safety, 109: 123-132.
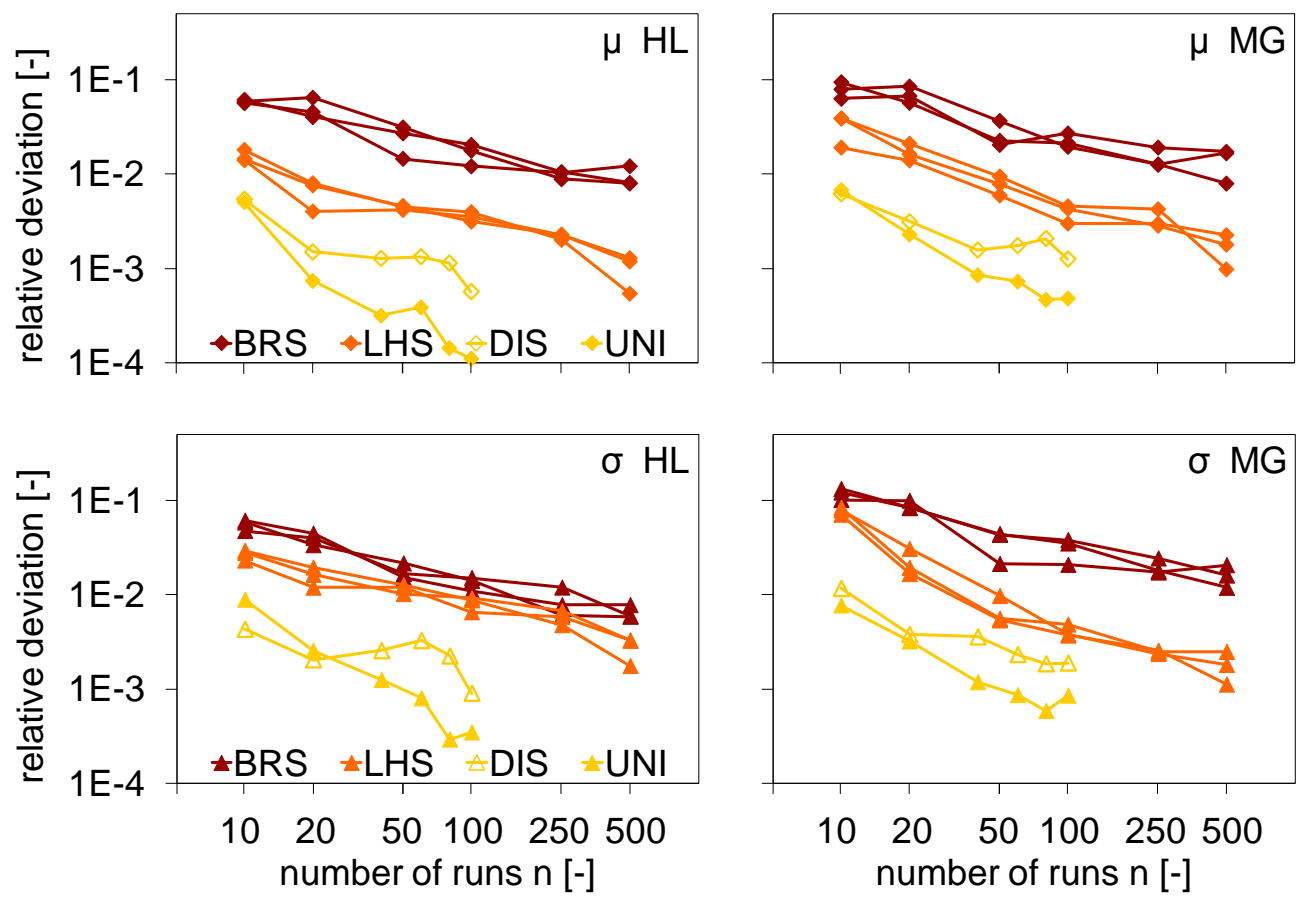

FIG 8. Normalised internal standard deviations for all series of ten $n$-run MonteCarlo sets.
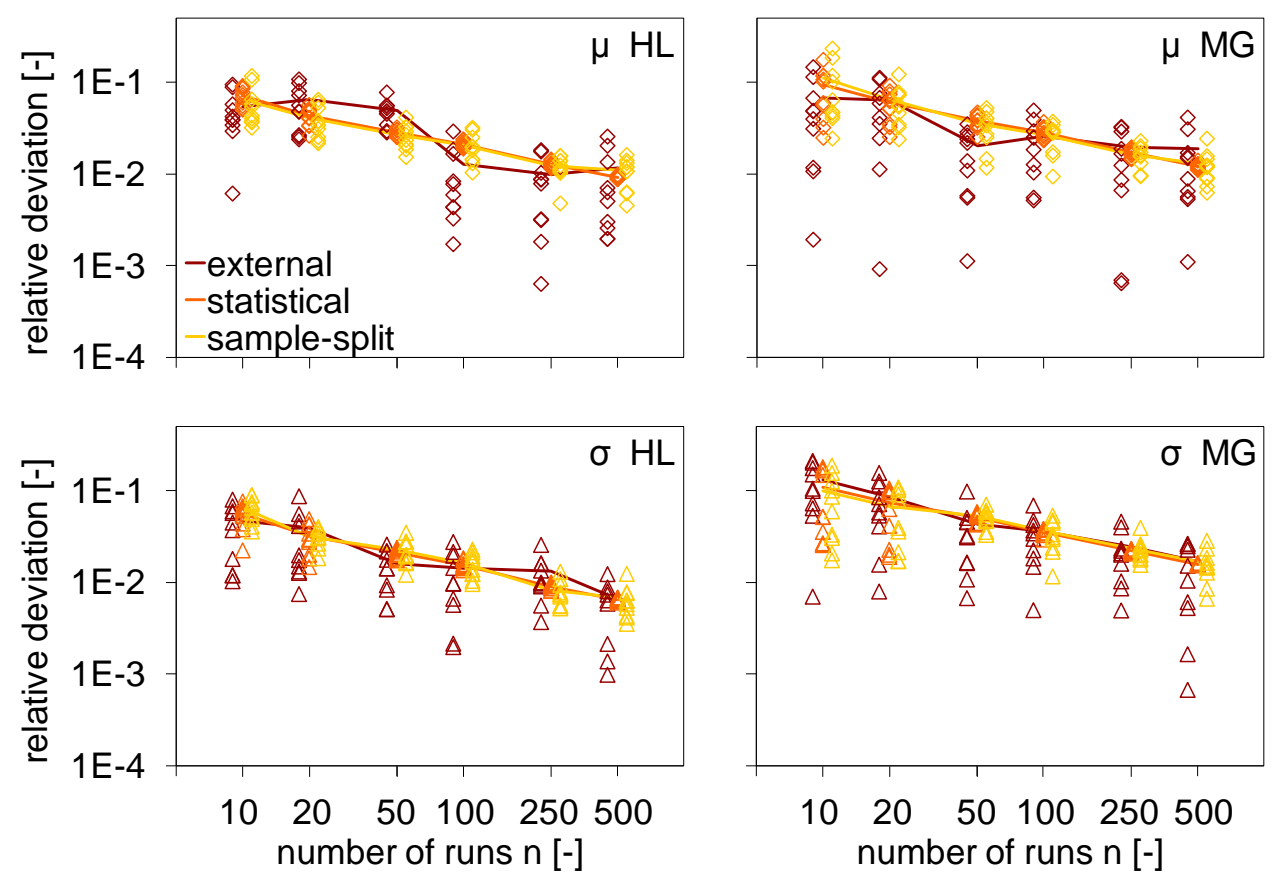

FIG 9. Normalised external deviations and normalised internal standard deviations (statistical and sample-split) for one BRS series of ten $n$-run Monte-Carlo sets. Hollow markers depict the normalised deviations, lines show their root-mean-squares. 
Postprint: Janssen H. 2013. Monte-Carlo based uncertainty analysis: Sampling efficiency and sampling convergence, Reliability Engineering \& System Safety, 109: 123-132.
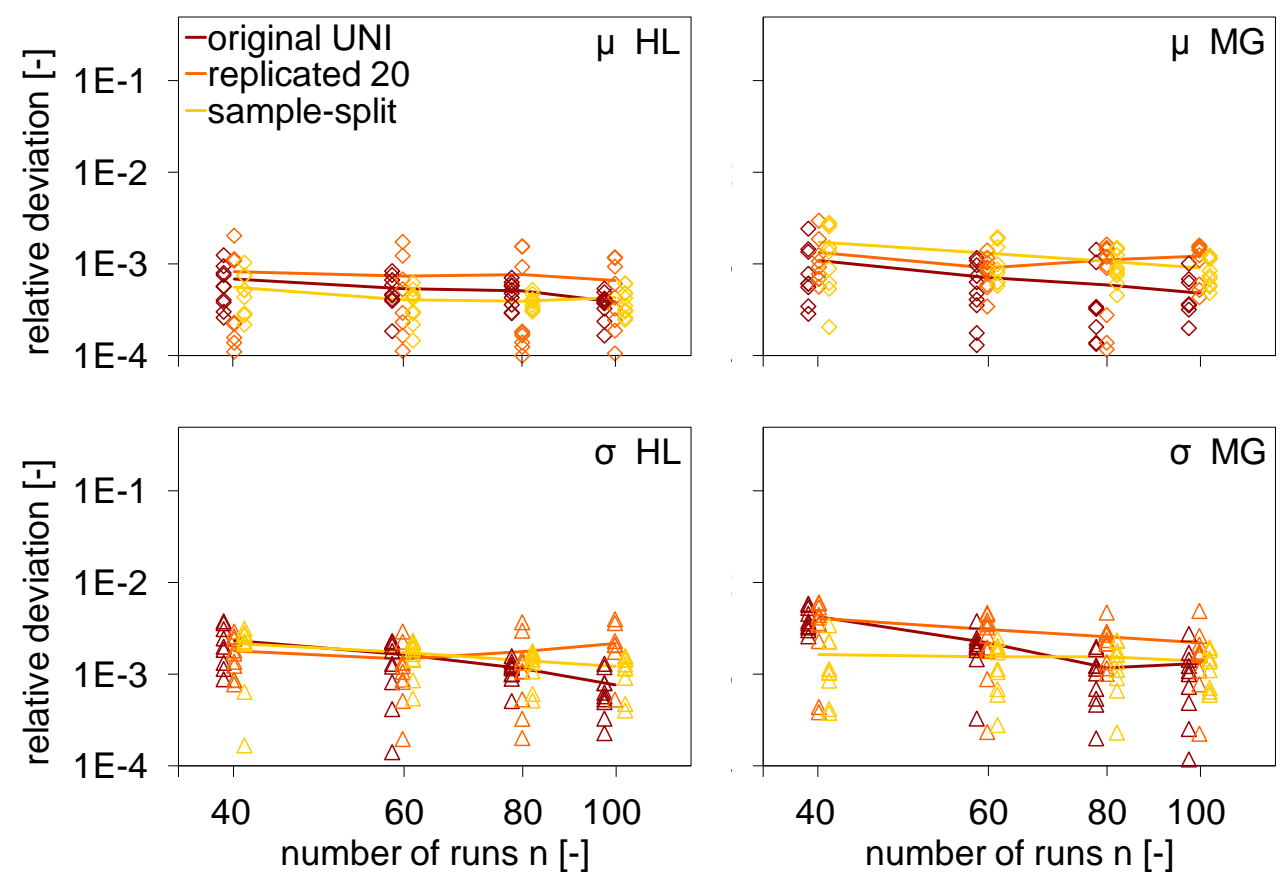

FIG 10. Normalised external deviations and normalised internal standard deviations (sample-split) for the original UNI results and the replicated-20 UNI results. Hollow markers depict the normalised deviations, lines show their root-meansquares.
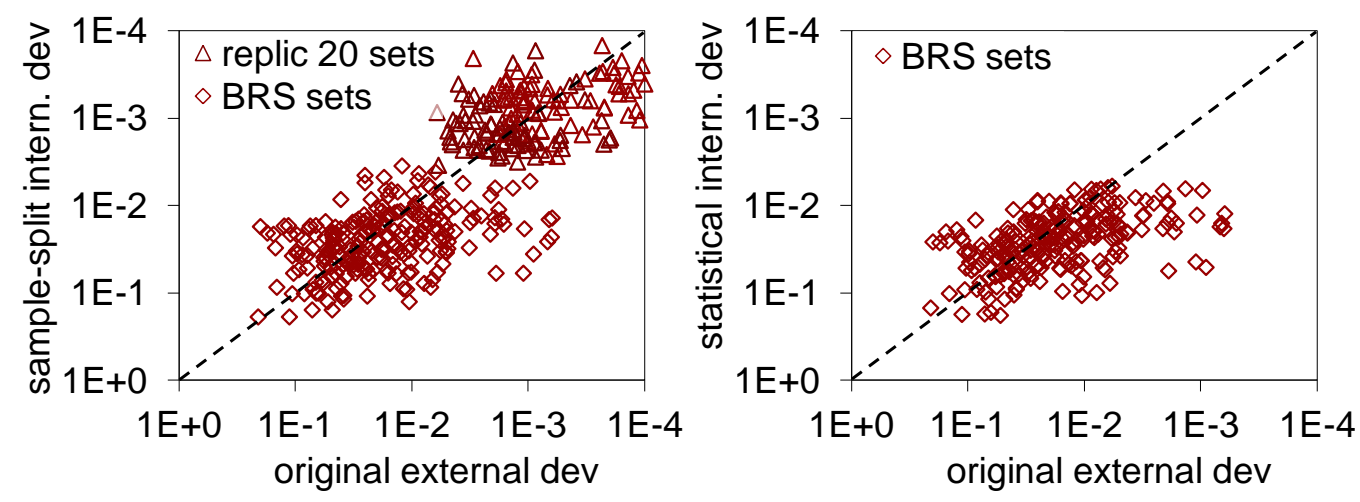

FIG 11. Comparison of normalised external deviations and normalised internal deviations (from sample-splitting) for separate $n$-run sets from the BRS and replicated UNI investi-gation (left); comparison of normalised external deviations and normalised internal devi-ations (from sampling statistics) for separate n-run sets from the BRS investigation (right). 
Postprint: Janssen H. 2013. Monte-Carlo based uncertainty analysis: Sampling efficiency and sampling convergence, Reliability Engineering \& System Safety, 109: 123-132.
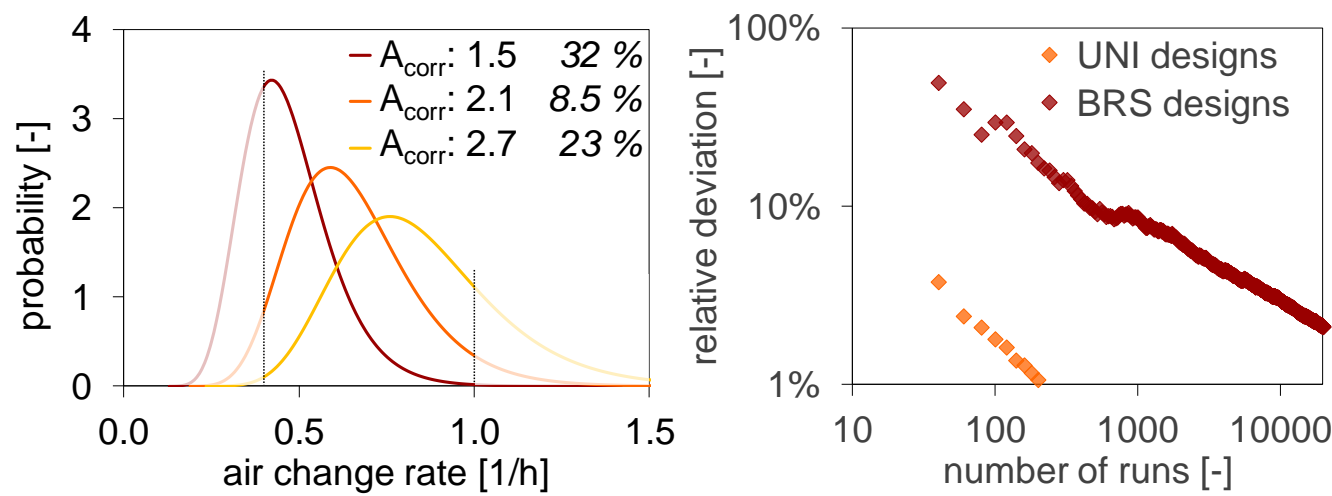

FIG 12. Resulting ACH distributions for $A_{\text {corr }} 1.5,2.1,2.7$, and the related probabilities of unfit ventilation (left); comparison of the convergence of the Monte Carlo analyses using $\mathrm{UNI}$ and BRS designs for the optimal $\mathrm{A}_{\text {corr }} 2.1$ (right). 